\title{
The IMAGES Database
}

The objectives of IMAGES (International Marine Global Changes Study) are to quantify climate and chemical variability of the ocean on time scales of oceanic and cryospheric processes; to determine their sensitivity to identified internal and external forcing, and to determine the oceans' role in controlling atmospheric $\mathrm{CO}_{2}$ (Pisias et al., IMAGES Science and Implementation plan, PAGES workshop report 94-3). To reach that objective, data acquisition and synthesis are major priorities, including coordination of international coring cruises. Four cruises have been completed on the Marion DuFresne:

- IMAGES I, MD101; North Atlantic Ocean and Norwegian Sea, 1995.

- IMAGES II, NAUSICAA; Namibia Angola upwelling system and Indian/Atlantic connection, 1996.

- IMAGES III, IPHIS I \&II; south of Australia and south-north transect from New Zealand to China Sea, 1997.

- IMAGES IV; Indonesian Archipelago, western Pacific and East China Sea, 1998.

The 1999 IMAGES $\mathrm{V}$ cruise is planned to retrieve annual to centennial resolution cores along a $\mathrm{N}-\mathrm{S}$ transect in the western and eastern parts of the North Atlantic, and the Greenland/

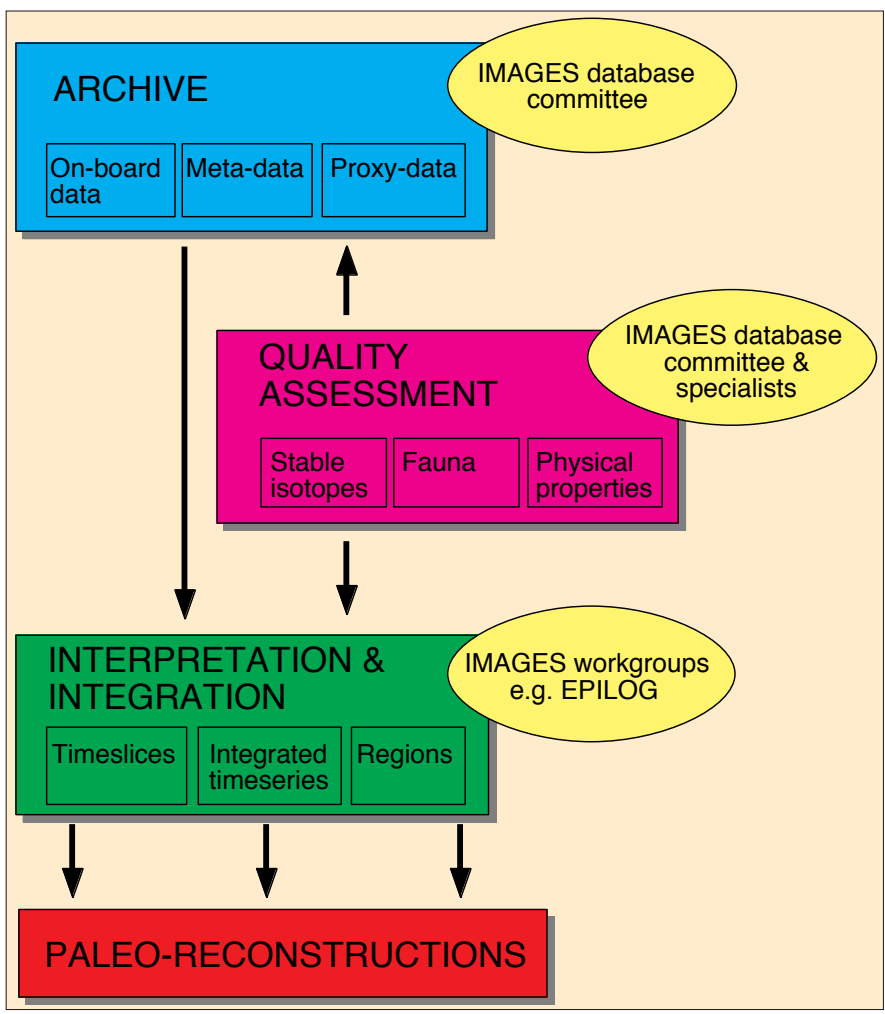

Norwegian Seas. IMAGES data are, however, not only data collected during IMAGES supported Marion DuFresne cruises. The goals will only be reached if we also use high quality marine paleoproxy data from other research programmes consistent with IMAGES philosophy and goals.

The goals demand also that the collected data are archived and data exchange is made possible from the first to the last phase of research. Finally, the ultimate results should be freely accessible for the entire scientific community. Therefore IMAGES decided to form a committee responsible for data management, consisting of Sacha De Rijk (chair), Dave Anderson (World Data Center advisor) and Frank Rack (physical properties advisor). The IMAGES database will be designed and compiled in close co-operation with the World Data Center at Boulder, USA (http:// www.ngdc.noaa.gov) and existing database efforts from various laboratories and agencies (e.g. the German PANGAEA information system, http:// www.pangaea.de).

One of the major features of the IMAGES database will be the introduction of data quality assessment. The archiving and interpretation/integration of data are the two other components of

Schematic outline of the structure of the planned IMAGES database the database (see figure). Data will be archived using protocols already established for other PAGES tasks and activities, and distributed freely over the Internet (http://www.images.cnrs-gif.fr).

Quality assessment is key to the success of the project, as IMAGES' aim is to gather high quality and high resolution data. With the help of specialists we will formulate a set of criteria to be applied to each data set. Criteria for stable isotopes, for example, will include details on calibration, number of specimens analysed, and preparation methods used. A quality index can then be attached to each data set in the IMAGES database. In other words, no data set will be excluded a priori from the subsequent interpretation and integration stage.

Interpretation and integration of the proxy data will be performed by various workgroups focusing on specific timeslices, methods or regions. One such workgroup (EPILOG) has already begun to re-evaluate the Last Glacial Maximum, allowing integration of paleodata into numerical climate modelling experiments (e.g. PMIP project). Additional reconstructions will be similarly derived from major IMAGES working groups (e.g. SEAMONS; Asian monsoon variability).

\section{Sacha De Rijk and Laurent Labeyrie}

Laboratoire des Sciences du Climat et de

I'Environnement, Laboratoire mixte CNRS-CEA, Gif sur Yvette, France

rijk@|sce.cnrs-gif.fr

labeyrie@|sce.cnrs-gif.fr

\section{David M. Anderson}

World Data Center for Paleoclimatology, Boulder, CO, USA

dma@ngdc.noaa.gov
The joint PAGES/SCOR marine program IMAGES (International Marine Global Change Study) provides travel grants for young scientists from developing countries to attend major IMAGES related scientific meetings. Scientists interested in learning more about the IMAGES program, or applying for travel funding, should contact either their national IMAGES representative or the IMAGES secretariat. Further information, including a list of member nations and national representatives, can be found on the web at http://www.images.cnrs-gif.fr. 